\title{
Morbilidad por alteraciones de la Microbiota Intestinal en niños nacidos por cesárea
}

\section{Morbidity due to intestinal microbiota alterations in children born by cesarean section}

\author{
Cristian Alberto López Bernal ${ }^{1}$ Concepción Herrera Gutiérrez² ${ }^{2}$ Concepción Amador Ahumada ${ }^{3}$
}

Recibido para publicación: Mayo 082020 - Aprobado para publicación: Noviembre 252020

\begin{abstract}
RESUMEN
La cesárea representa un riesgo para la mujer y el neonato, y se ha asociado con patologías en la infancia. Objetivo. Determinar el comportamiento estadístico de asma, hiper-reactividad bronquial y diabetes en niños de hasta 12 años según la vía del nacimiento en Montería. Materiales y métodos. Estudio analítico y descriptivo, a partir de información de 275 encuestas a familiares de niños menores de 12 años atendidos por cualquiera de las causas de morbilidad en estudio en el Hospital San Jerónimo de Montería, se configuró una base de datos en Microsoft Excel y se anexó al paquete estadístico SPSS25. El análisis fue a partir de estadística descriptiva y de correlación con prueba Chi-cuadrado de Pearson, y Prueba " $Z$ ", determinando relación entre las enfermedades y la vía de nacimiento. Resultados. El 50,2\% de los niños fue de sexo masculino. Se observó relación estadísticamente significativa entre diabetes y la vía de nacimiento por cesárea ( $p$-valor $<0,05)$, no hubo relación con las enfermedades respiratorias. Conclusiones. La cesárea fue la vía de nacimiento predominante, relacionada de forma significativa con diabetes en la población de estudio.
\end{abstract}

Palabras clave: Cesárea, Diabetes, Asma.

\begin{abstract}
Cesarean section represents a risk for women and newborns, and has been associated with pathologies in childhood. Objective. To determine the statistical behavior of asthma, bronchial hyper-reactivity and diabetes in children up to 12 years old according to the route of birth in Montería. Materials and Methods. Analytical and descriptive study, based on information from 275 surveys of relatives of children under 12 years of age attended by any of the causes of morbidity under study at the San Jerónimo de Montería Hospital, a database was configured in Microsoft Excel and it was attached to the statistical package SPSS25. The analysis was based on descriptive statistics and correlation with Pearson's Chi-square test and " $Z$ " test, determining the relationship between diseases and the route of birth. Results. $50.2 \%$ of the children were male. A statistically significant relationship was observed between diabetes and the cesarean birth route ( $p$-value $<0.05)$, there was no relationship with respiratory diseases. Conclusions. Cesarean section was the predominant birth route, significantly related to diabetes in the study population.
\end{abstract}

Keywords: Caesarean section, Diabetes, Asthma.

Citación (Vancouver)

López Bernal C, Herrega Gutiérrez C, Amador Ahumada C. Morbilidad por alteraciones de la Microbiota Intestinal en niños nacidos por cesárea. Rev Avances en Salud; 2020. (4)2:29-40. doi: 10.21897/25394622.2344

(C) 2020. Universidad de Cordoba. Este es un artículo de acceso abierto distribuido bajo los términos de la licencia Creative Commons Attribution License, que permite el uso ilimitado, distribución y reproducción en cualquier medio, siempre que el autor original y la fuente se acreditan.

\footnotetext{
${ }^{1}$ Magister(c) en Salud Pública - Docente Universidad de Córdoba. Correspondencia: chrislober1@gmail.com

${ }^{2}$ M.Sc. Docente. Universidad de Córdoba. Colombia.

${ }^{3}$ M.Sc. Docente. Universidad de Córdoba. Colombia.
} 


\section{INTRODUCCIÓN}

La cesárea representa riesgos para la mujer y su hijo por nacer; algunos inherentes al procedimiento quirúrgico y al acto anestésico $(1,2,3)$, que pueden producir complicaciones no previstas durante el nacimiento (4). La teoría de la "Hipótesis de higiene extendida", establece que los niños que nacen por cesárea presentan tipos de colonización diferente de la microbiota intestinal, respecto a los que nacen por vía vaginal (5). Se ha descrito que el nacimiento por cesárea se asocia a función pulmonar deteriorada, asma, enfermedad inflamatoria intestinal, artritis juvenil y leucemia posiblemente por efecto de alteraciones en la microbiota intestinal debido a la no utilización del canal del parto $(6,7)$. La poca exposición a bacterias al momento del nacimiento, se convierte en un factor de riesgo para el desarrollo de diferentes patologías, por la influencia de estos microorganismos en el desarrollo inmunitario del neonato $(1,2)$.

Se entiende entonces, que los excesos de cesáreas constituyen un importante problema de salud pública (8), las patologías asociadas a la cesárea generan incrementos en el gasto sanitario y en los recursos destinados para la asistencia en salud de la población materno infantil (9). Por ello, la Organización Mundial de la Salud (OMS), refiere como tasa ideal de cesáreas no mayor al 15\% (10), sin embargo, las tasa en Latinoamérica superan el $40 \%$. Destacan países como Costa Rica y Brasil con las mayores frecuencias, seguidos por Venezuela, Chile, Colombia, Paraguay, Ecuador, México y Cuba $(11,12)$. En Colombia, la proporción es del 46\% (11), en el departamento de Córdoba del $67,1 \%(13,14)$, y en Montería del $74,8 \%$ (15). Esta realidad local requiere estrategias urgentes para su reducción (12), pues la práctica de cesáreas no se ha acompañado de una mejoría de los índices de morbimortalidad perinatal (16-20).
La cesárea no garantiza seguridad para la madre, ni para el neonato; puesto que expone al recién nacido a morbilidades asociadas a este procedimiento $(21,22)$. Si bien, en la ciudad de Montería existen datos estadísticos respecto de cesáreas vs partos vaginales, no se han documentado los efectos patológicos inducidos por la microbiota intestinal en niños nacidos por cesárea o por parto vaginal. Por ello es necesario hacer un diagnóstico que permita ddeterminar el comportamiento estadístico de asma, hiper-reactividad bronquial y diabetes en niños de hasta 12 años nacidos mediante por esta vía.

\section{MATERIALES Y MÉTODOS}

\section{Tipo de estudio y muestra.}

Se desarrolló un estudio analítico, descriptivo y correlacional. En el cual se valoraron los antecedentes del nacimiento (vaginal o por cesárea) de niños hasta de 12 años de edad que consultaron por morbilidad en el Hospital San Jerónimo de Montería. La muestra de análisis se determinó a partir del número total de nacidos vivos (899 niños) en la institución en el año anterior. La muestra de niños menores de 12 años a los cuales se les aplicó la encuesta se obtuvo de la población de referencia (N: 899), se calculó el tamaño de muestra, $m=(270)$, utilizando la fórmula unificada para el cálculo de muestras de poblaciones finitas, utilizando la siguiente expresión:

$$
n=\frac{N Z^{2} \mathrm{PQ}}{E^{2}(\mathrm{~N}-1)+Z^{2} \mathrm{PQ}}
$$

El tamaño de muestra fue: $\mathrm{n}=275$; el error máximo admisible fue de $5 \%$ y el nivel de confianza del $95 \%$. Se procedió a aplicar las encuestas conforme con el número de elementos muestrales. El muestreo fue no probabilístico, la información se recolectó de forma progresiva durante un mes (octubre noviembre/2019) hasta completar el tamaño de muestra indicado. En la muestra de niños 
consultantes se tomó información de un niño con antecedente de nacimiento por parto vaginal por cada caso de niño nacido mediante cesárea (electiva o de urgencias) con el fin de estimar las diferencias estadísticas entre ambos grupos.

La información sobre los antecedentes del nacimiento de los niños atendidos, así como de los antecedentes familiares de las enfermedades relacionadas con alteraciones de la microbiota intestinal se tomó directamente de la fuente primaria mediante entrevista a la madre o cuidador, previo consentimiento informado.

Análisis de confiabilidad de la encuesta de morbilidad asociada a cesárea

Se realizó validación mediante prueba piloto con los familiares de 15 niños y validación estadística consistente en la verificación de la consistencia interna y fiabilidad de esta, para ello se utilizó el estadístico Alpha de Cronbach, cuyos valores oscilan entre 0 y 1. El cálculo se realizó mediante la siguiente formula:

$$
\alpha=\frac{k}{k-1}\left[1-\frac{\sum S_{i}^{2}}{S_{t}^{2}}\right]
$$

El proceso de validación se realizó utilizando el Software estadístico SPSS versión 25, lográndose los resultados que se muestran en las tablas 1 y 2 :

Tabla 1. Número de casos para validación.

Resumen del procesamiento de los casos

\begin{tabular}{ll|l}
\hline Casos & $\mathrm{N}$ & $\%$ \\
Válidos & 275 & 100 \\
Excluidosa & 0 & 0 \\
Total & 275 & 100
\end{tabular}

a. Eliminación por lista basada en todas las variables del procedimiento.

Fuente: Base de datos población encuestada.

En el procedimiento de validación se omitieron categorías de variables que no presentaron variabilidad durante el pilotaje pero que se incluyeron en el estudio. El resultado del coeficiente Alpha de Cronbach tuvo un valor de 0.833; (tabla 2). Teniendo en cuenta que el criterio de evaluación de fiabilidad es que los valores de Alpha de Cronbach sean superiores a 0.7; Se concluyó que el instrumento utilizado fue adecuado en cuanto a su consistencia interna y por tanto arrojó resultados confiables.

Tabla 2. Alpha de Cronbach del instrumento.

Estadísticos de fiabilidad

\begin{tabular}{ll}
\hline Alfa de Cronbach & $\mathrm{N}$ de elementos \\
0,833 & 14 \\
\hline
\end{tabular}

Fuente: Base de datos población encuestada.

La información compilada en la base de datos en Microsoft Excel $\circledast$, fue adicionada al Software estadístico SPSS25 IBM Corporation ${ }^{\circledR}$, con el cual se realizó análisis estadístico, calculándose los estadísticos descriptivos de acuerdo con cada variable y categoría, estimando la tasa de cesáreas, luego se procedió a establecer las relaciones y asociaciones estadísticas entre cada tipo de morbilidad y el antecedente del nacimiento; mediante prueba Chi-cuadrado de Pearson y la "prueba $Z$ ". La información fue presentada mediante tablas y gráficos acordes con el tipo de variables que contienen.

\section{RESULTADOS}

\section{Caracterización de la población de acuerdo con las variables sociodemográficas.}

En el segundo semestre del año 2019 se aplicó encuesta para caracterización sociodemográfica y epidemiológica de niños de hasta doce años de edad clasificados según la vía de nacimiento (vaginal o cesárea), la muestra calculada para la población fue de 270 niños, no obstante, se tuvo la oportunidad de valorar a 275. La población estuvo constituida en un $50,2 \%$ por niños y un $49,8 \%$ niñas, en cuanto a la edad, el promedio fue de 67,44 meses (aproximadamente 6 años), con rangos de edades que iban desde 0,0392 meses ( 1 día de nacido) hasta 155 meses (12 años), (tabla 3). 
Tabla 3. Distribución de acuerdo con la edad.

Edad del niño (meses)

\begin{tabular}{ll|ll}
\hline Edades & Frecuencia & Porcentaje & Porcentaje acumulado \\
$<17$ & 53 & 19,3 & 19,3 \\
$17-34$ & 22 & 8,0 & 27,3 \\
$35-51$ & 41 & 14,9 & 42,2 \\
$52-68$ & 17 & 6,2 & 48,4 \\
$69-86$ & 47 & 17,1 & 65,5 \\
$87-103$ & 21 & 7,6 & 73,1 \\
$104-120$ & 44 & 16,0 & 89,1 \\
$121-138$ & 17 & 6,2 & 95,3 \\
$139+$ & 13 & 4,7 & 100,0 \\
Total & 275 & 100,0 & \\
\hline
\end{tabular}

Fuente: Base de datos población encuestada en la E.S.E Hospital San Jerónimo de Montería.

Respecto al grupo étnico, el $65 \%$ de los niños se categorizó en la etnia mestiza, 6,2\% blancos, 3,3\% afrodescendientes, $1,5 \%$ indígena; en contraste el $23,6 \%$ no identificó el grupo étnico al que pertenecía. En cuanto al grupo poblacional al que pertenecía la familia, el $26,2 \%$ de las familias estaban en condición de desplazados, en contraste, el $70,9 \%$ manifestó no pertenecer a los grupos poblacionales de riesgo relacionados en la encuesta o no saber a cuáles de esos grupos pertenecía. En lo correspondiente al nivel socioeconómico, el $95,3 \%$ de las familias a las que pertenecían los niños eran de bajo ingresos pertenecientes al estrato 1 .

\section{Caracterización epidemiológica de la población de estudio.}

Independientemente de la vía de nacimiento, se observaron anomalías congénitas en algunos de los niños consultantes. La distribución de frecuencias mostró que, de los 275 niños, el $2,2 \%$ presentó problemas cardiacos, con igual frecuencia problemas respiratorios. En general, el $11,6 \%$ de los niños nació con algún tipo de malformación o enfermedad. Para contrastar lo hallado en enfermedades congénitas, se indagó con los padres o cuidadores de los menores en cuanto a antecedentes familiares de las patologías en estudio, con el fin de poder establecer las posibles asociaciones entre la enfermedad y la vía del nacimiento, en la figura 1 describen las frecuencias de ocurrencia de cada patología. Destaca que en 15,6\% de los niños se habían presentado antecedentes familiares de Asma, en otro $32 \%$ antecedentes de diabetes, siendo esta la patología de mayor frecuencia.

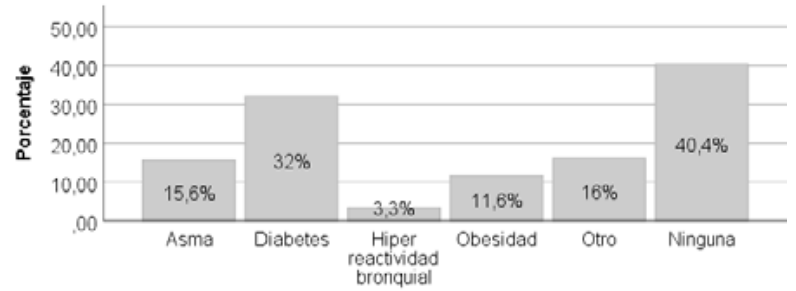

Gráfica 1. Antecedentes familiares de enfermedad.

Fuente: Base de datos población encuestada en la E.S.E Hospital San Jerónimo de Montería. 
De igual forma se indagó con los padres de los niños si los menores habían sido diagnosticados en algún momento del curso de su vida con alguna patología, inicialmente se observó la caracterización de la vía de nacimiento, y se encontró que $56,4 \%$ de los niños nacieron por cesárea y $43,6 \%$ por vía vaginal.

Tabla 4. Patologías frecuentes en la población de estudio de acuerdo con la vía de nacimiento.

Vía de nacimiento

\begin{tabular}{lr|r}
\hline Patología o signo clínico & Cesárea & Vaginal \\
Asma & $7,1 \%$ & $4,2 \%$ \\
Hiperreactividad bronquial & $3,9 \%$ & $0,8 \%$ \\
Obesidad & $1,3 \%$ & $1,7 \%$ \\
Otro & $11,6 \%$ & $10,8 \%$ \\
\hline
\end{tabular}

Fuente: Base de datos población encuestada

En el análisis bivariado se observó que el tipo de enfermedad más frecuente en los niños nacidos por cesárea fue el asma con el 7,1\% de los casos, seguido por el signo clínico híperreactividad bronquial 3,9\% (tabla 4 ).

\section{Comparación estadística de la presencia o ausencia de patologías de acuerdo con la vía de nacimiento.}

Se realizó comparación de "tipo inferencias" mediante la "prueba $Z^{\prime}$ " para proporciones utilizando el software SPSS25. Se le llamó $p 1$ a la proporción de niños nacidos por cesárea que han presentado una patología o un signo clínico particular, y a la proporción de niños nacidos mediante parto vaginal que han presentado esa misma patología, entonces la "prueba $Z$ " es una herramienta de la inferencia estadística para contrastar las hipótesis de que dichas proporciones son iguales o no:

$$
H_{0}: P_{1}=P_{2} \quad \text { vs } \quad H_{1}: P_{1} \neq P_{2}
$$

Inicialmente se realizó una prueba Chi-cuadrado para determinar si la presencia de enfermedades al momento del nacimiento dependía del tipo de parto. Los resultados de esta prueba en la tabla 5 , muestran un único valor $($ Sig. $<0.05$ ) ) correspondiente a la diabetes, lo cual indica una posible dependencia entre la presencia de esta diabetes juvenil y el parto por cesárea.

Tabla 5. Prueba Chi-cuadrado de Pearson: enfermedad vs vía de nacimiento. 


\begin{tabular}{ll|l}
$\begin{array}{ll}\text { Patología o signo clínico } \\
\text { Estadísticos }\end{array}$ & \multicolumn{1}{c}{ Estadísticos } & \multicolumn{1}{c}{ Vía de nacimiento } \\
\hline Asma & Chi-cuadrado & 0,171 \\
& df & 1 \\
Diabetes & Sig. & 0,679 \\
& Chi-cuadrado & 4,794 \\
Hiper-reactividad bronquial & df & 1 \\
& Sig. & $0,029 *$ \\
& Chi-cuadrado & 0,002 \\
\hline \multirow{2}{*}{ Obesidad } & Sig. & 1 \\
& Chi-cuadrado & $0,960 a$ \\
\hline Otro & df & 2,259 \\
& Sig. & 1 \\
\hline & Chi-cuadrado & 0,133 \\
\hline & df & 0,158 \\
\hline
\end{tabular}

Fuente: Base de datos población encuestada.

De igual forma, se realizó la prueba que le dio soporte a la prueba anterior, puesto que mediante esta prueba se hallaron diferencias significativas entre las proporciones de niños con diabetes, observándose que en los niños nacidos por cesárea la diabetes se presentó en una proporción que es significativamente mayor que en aquellos nacidos por parto vaginal (tabla 6).

Tabla 6. Prueba $Z$ enfermedad vs vía de nacimiento.

\begin{tabular}{|c|c|c|c|c|c|}
\hline \multirow{3}{*}{$\begin{array}{c}\text { Patología o signo } \\
\text { clínico }\end{array}$} & \multirow{3}{*}{ presencia } & \multicolumn{2}{|c|}{ Cesárea } & \multicolumn{2}{|r|}{ Vaginal } \\
\hline & & \multicolumn{2}{|c|}{ (A) } & \multicolumn{2}{|r|}{ (B) } \\
\hline & & Recuento & $\begin{array}{c}\% \text { de } \mathrm{N} \\
\text { columnas }\end{array}$ & Recuento & $\%$ de $N$ columnas \\
\hline \multirow[t]{2}{*}{ Asma } & No & 132 & $85,2 \%$ & 100 & $83,3 \%$ \\
\hline & $\mathrm{Si}$ & 23 & $14,8 \%$ & 20 & $16,7 \%$ \\
\hline \multirow[t]{2}{*}{ Diabetes } & No & 97 & $62,6 \%$ & $90(\mathrm{~A})$ & $75,0 \%$ \\
\hline & $\mathrm{Si}$ & $58(\mathrm{~B})$ & $37,4 \%$ & 30 & $25,0 \%$ \\
\hline \multirow{2}{*}{$\begin{array}{l}\text { Hiper-reactividad } \\
\text { bronquial }\end{array}$} & No & 150 & $96,8 \%$ & 116 & $96,7 \%$ \\
\hline & $\mathrm{Si}$ & 5 & $3,2 \%$ & 4 & $3,3 \%$ \\
\hline \multirow[t]{2}{*}{ Obesidad } & $\mathrm{No}$ & 133 & $85,8 \%$ & 110 & $91,7 \%$ \\
\hline & $\mathrm{Si}$ & 22 & $14,2 \%$ & 10 & $8,3 \%$ \\
\hline
\end{tabular}

Fuente: Base de datos población encuestada. 


\begin{tabular}{|c|c|c|c|c|c|}
\hline \multirow{3}{*}{$\begin{array}{c}\text { Patología o signo } \\
\text { clínico }\end{array}$} & \multirow{3}{*}{ presencia } & \multicolumn{2}{|c|}{ Cesárea } & \multicolumn{2}{|r|}{ Vaginal } \\
\hline & & \multicolumn{2}{|c|}{ (A) } & \multicolumn{2}{|r|}{ (B) } \\
\hline & & Recuento & $\begin{array}{c}\% \text { de } N \\
\text { columnas } \\
\end{array}$ & Recuento & $\%$ de $\mathrm{N}$ columnas \\
\hline \multirow[t]{2}{*}{ Otro } & No & 129 & $83,2 \%$ & 102 & $85,0 \%$ \\
\hline & $\mathrm{Si}$ & 26 & $16,8 \%$ & 18 & $15,0 \%$ \\
\hline
\end{tabular}

Los resultados se basan en pruebas bilaterales. Para cada pareja de significación, la clave de la categoría con la proporción de columna menor aparece en la categoría con la proporción de columna más grande. Nivel de significación para las letras mayúsculas (A, B, C): ,05a

a. Las pruebas se ajustan para todas las comparaciones por parejas dentro de una fila de cada subtabla más interna utilizando la corrección Bonferroni.

Fuente: Base de datos población encuestada.

De igual forma se contrastó la presencia de enfermedades en los niños nacidos por cesárea con los nacidos por parto vaginal, considerando el tiempo desde el nacimiento hasta la actualidad. Se realizó el procedimiento como en el caso anterior usando la prueba
Chi-cuadrado, y se encontró que no existía relación de dependencia entre la presencia de las enfermedades en estudio y el tipo de parto en esta muestra de 275 individuos puesto que el valor de significancia fue mayor a 0,05 en todos los casos (tabla 7).

Tabla 7. Prueba Chi-cuadrado de Pearson: comparación historial patológico.

\begin{tabular}{ll|r}
\multicolumn{1}{c|}{$\begin{array}{c}\text { Patología o signo clínico } \\
\text { Estadísticos }\end{array}$} & \multicolumn{1}{c}{ Estadísticos } & Vía de nacimiento \\
\hline Asma & Chi-cuadrado & 1,060 \\
& df & 1 \\
Digabetes & Chi-cuadrado & 0,303 \\
& df & . \\
Hiper-reactividad bronquial & Sig. & Chi-cuadrado \\
& df & 2,516 \\
& Sig. & 1 \\
Obesidad & Chi-cuadrado & $0,113 a$ \\
& df & 0,067 \\
Otro & Sig. & 1 \\
& Chi-cuadrado & $0,796 a$ \\
& df & 0,041 \\
\hline
\end{tabular}

Los resultados se basan en filas y columnas no vacías en cada subtabla más interna.

Fuente: Base de datos población encuestada. 


\section{DISCUSIÓN}

El marcado incremento anual del número de cesáreas electivas y el impacto de la vía de nacimiento en la salud de los niños es un tema que ha ido adquiriendo gran relevancia a nivel mundial (23). Si bien, no hay duda que las cesáreas son procedimientos que salvan vidas de mujeres y recién nacidos; es preciso señalar que este tipo de intervenciones se deben practicar cuando haya suficientes razones clínicas que la justifiquen, dadas las posibles consecuencias negativas de este procedimiento para la salud materno-infantil.

Conforme con esto, la OMS ha establecido una tasa ideal de cesáreas no mayor al $15 \%$, pero en Colombia la proporción de cesáreas gira en torno al $46 \%$; en Córdoba y Montería las cifras son alarmantes, del $67 \%$ y $74 \%$ respectivamente (11), por ello, resultó relevante intervenir con acciones prácticas y de investigación que permitieran identificar la relación entre algunas patologías que pueden presentar los niños menores de 12 años debidas a

las alteraciones de la microbiota intestinal que suceden en los partos por cesárea.

La edad promedio de los niños con morbilidad atendida fue 6 años, con predominio de varones, en su mayoría procedentes de Montería, pertenecientes a familias de bajo ingreso económico, la mayoría de los padres de estos se dedicaban a trabajo informal. Estos resultados desde la perspectiva de salud pública son relevantes puesto que ponen de manifiesto las condiciones de alta vulnerabilidad de la población infantil de la región. Condición que potencializa su efecto en la salud de esta población si se asocia con la vía de nacimiento, que en este estudio la forma predominante fue cesárea en el $56,4 \%$ de los casos.

Respecto a esta asociación, Sánchez (4) y Sadler (5), afirman que son más altas las tasas de infecciones gastrointestinales, de las vías respiratorias y sensibilización a alimentos alergénicos en los primeros años de vida de niños nacidos por cesárea en comparación con aquellos que nacen por la vía vaginal. Hipótesis que guarda relación con lo hallado en el presente estudio, en este se pudo constatar que los niños nacidos por vía vaginal no presentaron patologías en porcentajes más altos $(82,5 \%)$, que aquellos que nacieron por cesárea $(76,8 \%)$. Es decir, hubo una diferencia porcentual de $5,7 \%$ de no desarrollar enfermedad por haber nacido por la vía vaginal.

La afecciones respiratorias predominaron en nacidos por cesárea, en estos, el asma, la hiperreactividad bronquial y las enfermedades infecciosas fueron comunes, estos hallazgos concuerdan con lo descrito por Peters et al (6), quienes afirman que existe evidencia para plantear que el nacimiento por cesárea está asociado a un aumento en el número de casos de hipotermia, disfunción pulmonar, alteraciones del metabolismo y de presión sanguínea a corto plazo, y a mediano y largo plazo se asocia como factor de riesgo para que los niños desarrollen asma, diabetes tipo 1 , obesidad, enfermedad inflamatoria intestinal, artritis juvenil y leucemia.

Respecto a las causas que relacionan estas enfermedades con la vía de nacimiento, particularmente con la cesárea, Baeza y Chan (1) han destacado la diferente colonización a la que se expone el recién nacido conforme con la vía de nacimiento, que para el caso (según los autores), el tránsito fetal a través del canal vaginal no estéril facilita una contribución materna de Microbiota bacteriana al intestino neonatal, se aportan a este, bacterias de los géneros Lactobacillus spp., Prevotella spp., Sneathia spp. Contrariamente, en el proceso estéril de una cesárea, el neonato es colonizado con bacterias de la institución hospitalaria y de la piel de la madre, bacterias de los géneros Staphylococcus spp., Corynebacterium spp., Propionibacterium spp., las cuales no logran fortalecer inmunológicamente al recién nacido, sino que son un factor de enfermedad.

Serrano y Harris (23), sostienen que "la siembra inicial de microbiota en el neonato se da luego de la rotura del saco amniótico y proveniente 
de la microbiota oral, intestinal, vaginal y del tracto urinario de la madre, los recién nacidos son inoculados en su paso por el canal del parto (transmisión vertical), posteriormente, debido a la interacción con el medio (transmisión horizontal) se establece una composición más estable de la microbiota a la edad de dos años, por esto la cesárea es un procedimiento que promueve la disminución en la riqueza y diversidad de la microbiota, lo cual se ha asociado a un mayor riesgo ante diferentes enfermedades".

Sin embargo, estas teorías no fueron sometidas a prueba en la presente investigación, debido a que no se realizó un estudio experimental que permitiera caracterizar la microbiota intestinal de los recién nacidos para determinar las asociaciones de causalidad entre la microbiota intestinal y su relación con el desarrollo de enfermedades en el neonato de acuerdo con la vía de nacimiento, de manera que las asociaciones establecidas fueron solo de tipo estadístico, buscando una explicación cercana a las condiciones reales.

En las pruebas de significancia, no se hallaron asociaciones estadísticamente significativas entre el desarrollo de asma, hiperreactividad bronquial y obesidad con la vía de nacimiento de la población estudiada, en estos casos los valores fueron superiores que 0,05 (Chi-cuadrado de Pearson); no obstante, la asociación fue significativa con diabetes, resultado corroborado mediante "prueba $z$ " con esta se hallaron diferencias significativas entre las proporciones de niños con diabetes, observándose que en los niños nacidos por cesárea esta enfermedad se presentó en una proporción que fue significativamente mayor que en aquellos nacidos por parto vaginal. Estos resultados concuerdan con lo descrito por Serrano y Harris (23), quienes sostienen que alteraciones en las interacciones tempranas entre los microorganismos comensales y el huésped han sido asociadas con efectos a largo plazo en el desarrollo de la inmunidad y del metabolismo conllevando a trastornos como la obesidad y diabetes.
Los resultados difieren de lo hallado por Sadler (5), quien, en un estudio de cohorte de base poblacional realizado en Australia, en el cual no se no mostró asociación entre cesárea y diabetes, pero mostró que los niños nacidos por cesárea presentan un riesgo superior de padecer infecciones respiratorias y otros tipos de infecciones, dermatitis atópica y desorden metabólico hasta los cinco años, en comparación con niños nacidos por vía vaginal.

Por otro lado, al indagar sobre los antecedentes familiares de las patologías en estudio, se observó con mayor frecuencia los antecedentes de asma, obesidad y diabetes, siendo esta ultima la de mayor frecuencia, lo cual no desestima la asociación diabetes vs nacimiento por cesárea descrita anteriormente, puesto que la diabetes fue antecedente familiar común en los niños nacidos por ambas vías, cesárea y parto vaginal.

Adicionalmente, aunque el asma y la hiperreactividad bronquial no presentaron significancia estadística respecto a la vía de nacimiento, estas patologías fueron más frecuentes en los niños nacidos por cesárea. Los hallazgos en conflicto pueden deberse a los métodos estadísticos utilizados, o tal vez a diferencias en las características sociodemográficas, y la falta de un abordaje experimental que permitiese establecer una relación entre microbiota intestinal en neonatos nacidos por cesárea y parto vaginal, con enfermedades respiratorias. Furzan (24) sugiere que las afecciones respiratorias están más relacionadas con morbilidad neonatal asociada a la cesárea, que va desde procesos benignos de taquipnea transitoria a casos graves de insuficiencia respiratoria hipóxica y muerte.

Por su parte, Arboleya (25) describe que uno de los factores que contribuyen al desarrollo normal de la microbiota intestinal y por ende al crecimiento de niños sanos, es el nacimiento a término por vía vaginal y que reciben lactancia materna exclusiva, y Gil (26) plantea la perspectiva en cuanto a diagnóstico 
y tratamiento de otras patologías, que de seguir constatándose la relación entre las vías de nacimiento con el desarrollo de alergias, inmunodeficiencias, y obesidad, se podría lograr la erradicación de las mismas no mediante la vacunación, sino mediante el trasplante directo de la microbiota o tratamientos probióticos o prebióticos, estos y muchos otros aspectos son motivantes para seguir desarrollando investigaciones en esta área.

\section{CONFLICTO DE INTERÉS}

Los autores declaran no tener conflicto de interés.

\section{CONCLUSIONES}

No se hallaron relaciones estadísticamente significativas entre la vía de nacimiento y patologías como asma, obesidad o el signo clínico hiperreactividad bronquial en la población de estudio. Sin embargo, fue estadísticamente significativa la asociación entre diabetes y nacer por cesárea.

\section{REFERENCIAS}

1. Baeza M, Chan R. Nacimiento por cesárea y desarrollo de asma en escolares. Revista Mexicana de Pediatría. 2015; 82(4): 124128.

2. Aguilar R, Manrique G, Aisa L, Delgado L, González V, Aceituno L. Uso de la clasificación de Robson en un Hospital Comarcal de España para reducir la tasa de Cesáreas. Revista Chilena de Obstetricia y Ginecología. 2016; 81(2): 99-104.

3. El País [internet]; El uso abusivo de cesáreas en América Latina contradice a la OMS; 2013. Disponible en: http:// sociedad.elpais.com/sociedad/2013/03/15/ actualidad/1363388139_662271.html.

4. Sánchez, D. Impacto de La Cesárea Sobre la Salud Materna y Perinatal: Revisión Sistemática, Trabajo de grado.
Bogotá, Universidad del Rosario. 2012. Recuperado a partir de: http:// repository.urosario.edu.co/bitstream/ handle/10336/3191/52860332-2012.

5. Sadler M. Los efectos de la cesárea en la salud infantil: un asunto urgente. Revista Chilena de Pediatría. 2018; 89(4): 561-562.

6. Peters L, Thornton $C$, de Jonge $A$, et al. The effect of medical and operative birth interventions on child health outcomes in the first 28 days and up to 5 years of age: A linked data population-based cohort study. Birth 2018; 00: 1-11.

7. Sevelsted A, Stokholm J, Bonnelykke K, Bisgaard $\mathrm{H}$. Cesarean section and chronic immune disorders. Pediatrics 2015; 135 (1): 92-8.

8. Zuleta J, Quintero F, Quiceno A. Aplicación del modelo de Robson para caracterizar la realización de cesáreas en una institución de tercer nivel de atención en Medellín, Colombia. Revista Colombiana de Obstetricia y Ginecología. 2013; 64(2): 9099.

9. Consenso de la Federación Colombiana de Obstetricia y Ginecología (FECOLSOG) y la Federación Colombiana de Perinatología (FECOPEN). Racionalización del uso de la cesárea en Colombia. Revista Colombiana de Obstetricia y Ginecología [internet]. 2014. Vol. 65 No.2, pp 139-151. doi: http:// dx.doi.org/10.18597/rcog.62.

10. World Health Organization. Appropiate technology for birth. Lancet 1985; 2:436-7.

11. Puentes, E., Gómez, O., Garrido, F. Las cesáreas en México: tendencias, niveles y factores asociados. Salud Pública de México. 2004; 46 (1): 16-22.

12. El heraldo saludo [internet]. América Latina, la región con la mayor tasa de cesáreas del mundo: estudio. 2018. Recuperado de: https://www.elheraldo.co/salud/americalatina-la-region-con-la-mayor-tasa-decesareas-del-mundo-estudio-552718.

13. Colombia. Departamento Administrativo Nacional de Estadísticas (DANE). Informe de nacimientos por tipo de parto según departamento de ocurrencia y sitio del parto en el 2016. Consultado el 20 de 
dic de 2018 en https://www.dane.gov. co/index.php/estadisticas-por-tema/salud/ nacimientos-y-defunciones/nacimientos/ nacimientos-2016.

14. Colombia. Departamento Administrativo Nacional de Estadísticas (DANE). Informe Preliminar de nacimientos por tipo de parto según departamento de ocurrencia y sitio del parto en el 2018. Consultado el 20 de dic de 2018 en https://www.dane.gov. co/index.php/estadisticas-por-tema/salud/ nacimientos-y-defunciones/nacimientos/ nacimientos-2017.

15. Estadísticas de RUAF, Ministerio de Protección Social, información suministrada por la Secretaria de Salud Municipal, lunes 17 de diciembre de 2018.

16. Faneite P, Rivera C, Faneite J, Amato R. Cesárea y mortalidad perinatal. Incidencia y tendencia 42 años. Rev Obstet Ginecol Venez 2012; 72(3):145-151.

17. Tong L. Rhodes G, Smulian J, Demissie K, Wartenberg D, Lakota K. Physician cesarean delivery rates and risk-adjusted perinatal outcomes. Obstet Gynecol 2003; 101(6):1204-1212.

18. Larraz J, García J, Díez L, Fernández M, Paredes J. La aplicación de la medicina basada en la evidencia reduce la tasa de cesáreas. Prog Obstet Ginecol 2009; 52(8):427-436.

19. MacDorman M, Declercq E, Menacker F,
Malloy $\mathrm{MH}$. Neonatal mortality for primary cesarean and vaginal births to low-risk women: application of an "intention to treat" model. Birth 2008; 35(1):3-8.

20. Villar J, Carroli C, Zavaleta N, Donner A, Wojdyla D, Faundes A, et al. Maternal and neonatal individual risks and benefits associated with caesarean delivery: multicenter prospective study. BMJ 2007; 335(7628):1025-1036.

21. Robson M. Classification of caesarean sections. Fetal Matern Med Rev. 2001; 12:23-39.

22. Robson M, Scudamore I, Walsh S. Using the medical audit cycle to reduce cesarean section rates. Am J Obstet Gynecol. 1996; 174:199-205

23. Serrano C, Harris P. Desarrollo del microbioma intestinal en niños: impacto en salud y enfermedad. Revista Chilena de Pediatría. 2016; 87(3): 151-153.

24. Furzan J. Nacimiento por cesarea y pronóstico neonatal. Revista Archivos Venezolanos de Puericultura y Pediatria. 2014; 77(2):79-86.

25. Arboleya S. Estudio del establecimiento de la microbiota intestinal en neonatos y selección de probióticos para su modulación. 2014

26. Gil L, Catalina A. La microbiota, forjando el órgano invisible. MoleQla: 